\title{
Belphégor
}

Littérature populaire et culture médiatique

10-3 | 2011

Peter Pan

\section{Sophie Beaulé, Jean-Louis Trudel}

\author{
Amy J. Ransom
}

\section{OpenEdition}

Journals

Édition électronique

URL : http://journals.openedition.org/belphegor/419

DOI : 10.4000/belphegor.419

ISSN : 1499-7185

Éditeur

LPCM

Édition imprimée

Date de publication : 10 janvier 2011

\section{Référence électronique}

Amy J. Ransom, «Sophie Beaulé, Jean-Louis Trudel », Belphégor [En ligne], 10-3 | 2011, mis en ligne le 10 janvier 2013, consulté le 22 septembre 2020. URL : http://journals.openedition.org/belphegor/419 DOI : https://doi.org/10.4000/belphegor.419

Ce document a été généré automatiquement le 22 septembre 2020.

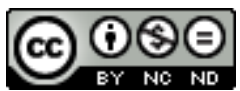

Belphégor est mis à disposition selon les termes de la Licence Creative Commons Attribution - Pas d'Utilisation Commerciale - Pas de Modification 4.0 International. 


\title{
Sophie Beaulé, Jean-Louis Trudel
}

\author{
Amy J. Ransom
}

\section{RÉFÉRENCE}

Sophie Beaulé, Jean-Louis Trudel, Québec, Les Éditions David, Voix didactiques 7, 384

pages, ISBN : 978-2-89597-092-7

1 Le premier livre consacré à l'étude d'un seul auteur du très actif milieu de la sciencefiction et du fantastique canadien-français, Jean-Louis Trudel par Sophie Beaulé, fait honneur à son sujet. Les Éditions David, dédiées à la publication d'auteurs francophones en dehors du Québec, offrent dans la série «Voix didactiques» des « monographies sur des auteurs de jeunesse et guides d'enseignement. » Cette étude du cycle de romans, Les Saisons de Nigelle (1997-2000), remplit bien cet objectif pédagogique sans pour autant décevoir le chercheur qui désire connaître l'œuvre de l'écrivain franco-ontarien. Trop souvent méprisé, le guide pédagogique se trouve racheté sous la main de Beaulé et ce travail offre un modèle pour d'autres ouvrages de référence dans sa structure et dans son style.

2 Figure de proue dans le mouvement de la «SFFCF, » Trudel est aussi l'ingénieur d'une série originale en ce que chaque roman des Saisons de Nigelle propose au lecteur un genre littéraire distinct: "mystère », science-fiction, fantastique épique (fantasy), et deux romans fantastiques, la plupart avec un « dispositif de lecture policière » selon Beaulé (p.61). C'est surtout cette originalité qui distingue le cycle de Nigelle d'autres séries pour la jeunesse et qui offre un surplus d'intérêt aux enseignants cherchant à présenter de façon rigoureuse les genres populaires aux étudiants. L'étude de Beaulé offre des outils documentaires essentiels : des définitions des genres en question, une analyse structurale de chaque roman, une étude des personnages et des thèmes clés, une quinzaine de tableaux analytiques aussi bien que des questions et des activités de réflexion.

3 Après une brève introduction qui présente le cas de l'importance de la série Nigelle et une biographie de son auteur, le travail se divise en deux parties, «Étude des Saisons de 
Nigelle » et un « Dossier » d'informations de soutien pour enrichir la lecture d'un ou de tous les romans de la série. La première partie propose un chapitre analytique à propos de chaque roman, structure utile pour ceux qui ne peuvent se référer qu'à l'un des cinq romans en question. Chacun de ces chapitres se divise encore en plusieurs sections, en commençant par un résumé de l'intrigue du récit. Ensuite, dans la section «Espace et temps ", Beaulé identifie un concept organisateur qui structure toute la série, celui des " sédiments" temporels, spatiaux et identitaires, dont nous traiterons plus tard. La description des personnages les insère au sein d'une analyse structurale à la Greimas et celle des thèmes souligne la préoccupation de la série avec les questions de la justice sociale, de la quête de la vérité, de la communication des valeurs, aussi bien que des thèmes typiques de la science-fiction et du fantastique comme la relation entre la réalité et l'imaginaire, et le rapport entre le Chaos, l'Ordre et des Portes vers d'autres mondes ou d'autres niveaux de l'existence. Une section très utile « Modalités et formes du récit» identifie le genre (quelquefois hybride) de chaque roman, et traite de questions d'intertextualité, de voix narrative et de style. Cette dernière section rehausse l'appréciation du lecteur de la qualité et de la complexité de ces «simples » romans de jeunesse. Enfin, une conclusion et des «Pistes de réflexion » closent chaque chapitre de la partie analytique. La conclusion générale à l'analyse de la série résume aussi la réception critique des romans à propos de laquelle Beaulé admet que «c'est auprès des critiques spécialisés en littérature pour la jeunesse et, surtout, en sciencefiction que le cycle a trouvé écho » (p. 315).

4 L'utilité du travail de Beaulé se voit non seulement dans sa structure claire et symétrique, mais aussi dans tous les soutiens pédagogiques tels que les tableaux compréhensifs pour la série (genres, personnages, composition comparée, etc.) et d'autres tels que des schémas actantiels greimassiens pour chaque roman. Le dossier, qui comprend une entrevue avec Jean-Louis Trudel et des informations (et des liens internet pour la poursuite indépendante des investigations sur ces matières de fond) sur des sujets relatifs aux romans, tels que la véritable géographie de Nogent-le-Rotrou et de sa région, les événements et les faits divers qui inspirent l'intrigue des romans, et les figures mythiques et légendaires auxquelles la série fait référence. Une liste d'autres lectures pour adolescents dans ces genres de fiction et une bibliographie terminent l'ouvrage.

5 Certains pourraient trouver à redire contre l'ouvrage par rapport à l'utilisation de sources en ligne pour le dossier, mais c'est ce genre de ressources qui va être le plus utile aux enseignants et à leurs étudiants qu'on le veuille ou non. Pour son analyse littéraire Beaulé renvoie son lecteur à des ouvrages théoriques de premier ordre aussi bien qu'à des manuels de référence tels ceux de la série "Que sais-je?». Parmi les repères théoriques mentionnés figurent les noms de Greimas, Genette, Eliade, Caillois, Bachelard et Suvin, sans oublier l'application à la littérature québécoise de ces théoriciens européens par Roland Bourneuf et Réal Ouellet, Richard Saint-Gelais et Yvon Allard parmi d'autres. Professeur agrégé, spécialiste du nouveau roman français aussi bien que de la science-fiction québécoise, Beaulé présente des éléments de la mythocritique, de l'analyse structurelle, et des études des genres de façon rigoureuse mais dans un langage clair et accessible aux enseignants de tous les niveaux aussi bien qu'aux étudiants du niveau secondaire et du cégep/premier cycle universitaire.

6 Ce qui m'impressionne dans la démarche de Beaulé est sa capacité de jeter de la lumière sur la qualité littéraire, intellectuelle et didactique de l'œuvre de Jean-Louis Trudel, et 
cela tout d'abord par l'identification de ce concept organisateur du cycle Nigelle les "sédiments, " spatiotemporels, identitaires et de l'imaginaire (p. 21). Il n'est pas surprenant que Trudel, qui est principalement connu comme écrivain de sciencefiction, ait exploité dans sa fiction un principe scientifique emprunté à la géologie, celui de la formation des couches de sédiments dans la création de certaines pierres. Ce principe informe aussi la pratique archéologique de fouiller dans les couches de terre superposées pour remonter de plus en plus profondément dans le temps. Beaulé analyse « le travail de sédimentation historique » (p. 33), c'est-à-dire des références au passé et au futur au sein d'un présent diégétique variable, depuis le « vrai » Moyen Âge à la Deuxième Guerre Mondiale, du tournant des $\mathrm{xx}^{\mathrm{e}}$ et $\mathrm{xxI}^{\mathrm{e}}$ siècles jusqu'au futur proche et à un Moyen Âge virtuel. Elle remarque comment la série souligne l'importance du temps par son choix de titres (les quatre premiers romans prennent leur nom d'une des quatre saisons et le dernier s'intitule Nigelle par tous les temps). Une étude des sédiments spatiaux va de pair avec l'analyse temporelle et Beaulé examine le jeu « cartographique » auquel se livrent les romans de Trudel et par lequel leur auteur engage son public à participer en mettant en scène des lieux réels à côté des lieux imaginaires. Ce jeu spatial entre le réel et l'imaginaire introduit encore les sédiments de l'imaginaire; chaque roman met en scène les questions du réel et de l'imaginaire, soit par le jeux de rôles virtuels poussé à leur extrême dans le roman de science-fiction (Un printemps à Nigelle), soit par la mise-en-scène d'un fantastique à la Todorov où l'on hésite entre une explication rationnelle ou une explication surnaturelle des événements insolites qui ont lieu (Un été à Nigelle), soit par la création du «Monde de Taurelaure ", un royaume de fées qui interagit avec un Moyen Âge "réaliste » où pourtant l' «ici-bas terrestre [est] entaché de surnaturel (magie, superstitions...)» (p. 35).

7 Cette étude de l'œuvre de Jean-Louis Trudel ne se recommande pas qu'aux enseignants, mais aussi à tous ceux qui veulent connaître de façon plus approfondie cet auteur franco-ontarien. De plus, elle offre à tout étudiant de la littérature de jeunesse ou des genres populaires des modalités de discours pour parler de façon savante de ces genres trop souvent marginalisés du canon. Dans sa bibliographie, l'auteure offre des ressources essentielles pour faire les premiers pas dans l'étude du polar, de la sciencefiction et du fantastique. Enfin, dans sa section «Pistes de réflexion » elle offre à tous les professeurs des modèles d'activités et de questions de discussion dans l'examen des textes littéraires aussi bien que des textes populaires. Jean-Louis Trudel par Sophie Beaulé nous présente un auteur et une série assez peu connus dans le monde universitaire et prouve qu'ils tiennent bien le coup sous son interrogatoire soutenu. 